\title{
Epithelioid Glioblastoma, A Newly Described Rare Variant of Common Tumor Describing Immunohistochemical Expression Pattern
}

\author{
Rakesh Kumar Gupta ${ }^{1 *}$, Neha Garg ${ }^{2}$, Vineeta V Batra ${ }^{1}$ and Lalit Garg ${ }^{3}$ \\ ${ }^{1}$ Departments of Pathology, G B Pant Institute of Postgraduate Medical Education and Research, New Delhi India. \\ ${ }^{2}$ Department of Pathology, Geetanjali Medical College and Hospital, Udaipur, Rajasthan, India. \\ ${ }^{3}$ Departments of Radiodiagnosis, G B Pant Institute of Postgraduate Medical Education and Research, New Delhi, India
}

\section{Dear Sir,}

Epithelioid glioblastoma (Ep-GBM) has recently been recognized as a distinct variant of glioblastoma (GBM), which is included in the 2016 revised World Health Organization (WHO) classification of central nervous system (CNS) tumors. ${ }^{[1]}$ Together with giant cell glioblastoma and gliosarcoma, it is kept under the broad category of IDH-wildtype glioblastoma. Ep-GBM is characterized by large cells with eccentric nuclei, vesicular chromatin, prominent nucleoli and abundant amount of eosinophilic cytoplasm. In addition, they may also show glandular, ductular and squamoid pattern expressing both GFAP and epithelial markers (cytokeratins) thus closely mimicking with the metastatic carcinomas. ${ }^{[2]}$ Hence, they should be carefully distinguished from metastatic carcinomas of unknown primaries. In contrast to typical GBM, they possess a higher predilection for children and younger adults, and usually present as superficial cerebral mass. Epithelioid GBMs frequently shows $B R A F-\mathrm{V} 600 \mathrm{E}$ mutation. ${ }^{[3]}$ Rhabdoid glioblastoma is a close histological mimicker of Ep-GBM, however it is differentiated by the loss of expression of INI-1. ${ }^{[4]}$

A 48-years old male presented to the Emergency Department of Neuro-surgery with the sudden onset of multiple episodes of vomiting, headache and weakness in the right half of body. On examination, patient was conscious, oriented with a normal Glasgow coma score. The power was $4 / 5$ in all the four limbs. Haemogram, serum electrolytes, kidney and liver functions were within normal limits. A Non contrast computer tomography scan was done which revealed a large intra-axial heterogenous hyperdense mass lesion with significant peri-lesional edema and midline shift (Figure 1A). The magnetic resonance imaging showed large intra-axial based mass lesion in left para-falcine region in parieto-occipital lobe showing CSF cleft surrounding the tumor brain interface with extensive peri-lesional white matter edema, intra- lesional hemorrhage and mass effect (Figure 1B-1F). A radiological diagnosis of high grade glial tumor with intra-lesional bleed was made. A left parieto-occipital flap craniotomy with complete excision of tumor was done.

Pathological findings: The tumor was comprised of large epithelioid cells arranged in sheets with perivascular clustering displaying eccentric nuclei with vesicular chromatin, prominent nucleolei and abundant amount of eosinophilic cytoplasm (Figure 2). Frequent bi and multinucleation, necrosis and atypical mitotic figures were noted. The cells showed diffuse positivity for GFAP, S-100, epithelial membrane antigen (EMA), CK20 and vimentin (Figure 3). Tumor showed retention of INI-1 expression, while IDH-1 was negative. BRAF-V600E mutation analysis was not performed due to unavailability of resources. Based on morphology and immunohistochemistry a final diagnosis of IDH-wild type Ep-GBM was made.

The first case of Ep-GBM was reported by Kepes et al followed by few subsequent case reports. Ep-GBM possesses a poor outcome with survival less than the median survival time of 14.6 months in conventional GBM. ${ }^{[5]}$ Most of the reported cases of Ep-GBM occurred denovo as a primary lesion. However, Alexandrescu et al showed that Ep-GBM and epithelioid pleomorphic xanthoastrocytoma (PXA) shares many molecular features such as BRAF-V600E mutation, wild type IDH, retained INI-1 expression and frequent loss of ODZ3 deletion. ${ }^{[6]}$ Similarly, Tanaka et al also described a case of Ep-GBM arising in a patient diagnosed with PXA 13-years after the treatment. ${ }^{[7]}$ Considering the above two examples it seems that Ep-GBMs are closely related with PXA which is a low grade glial tumor in comparison to conventional GBMs. However, since Ep-GBM has poor outcomes than typical GBM as well as uncertain response to temozolamide, this hypothesis is questionable and needs further evaluation. In our case, patient was referred for chemotherapy and later lost to follow-up. 


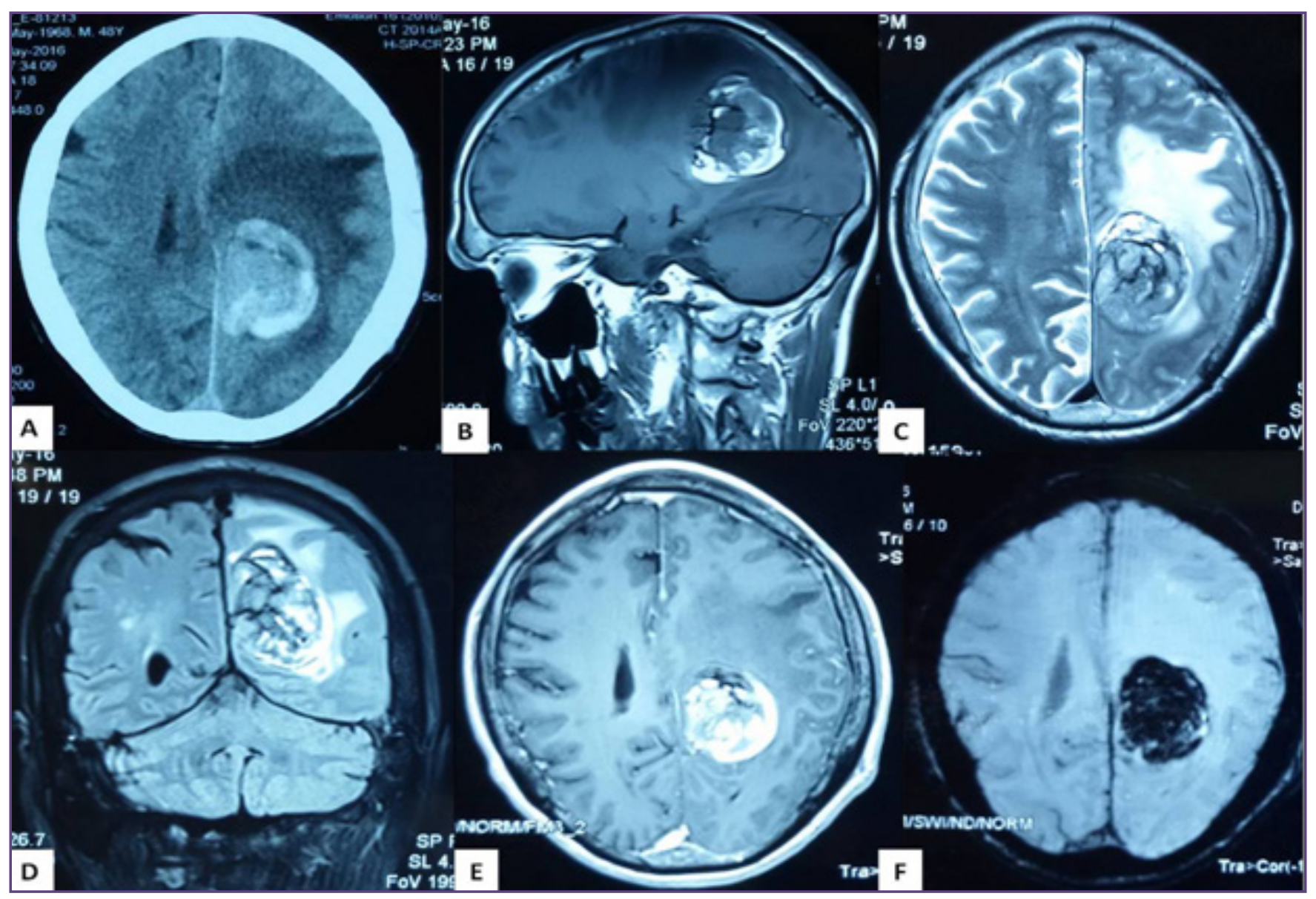

Fig. 1: (A) Axial NCCT image showing large intra-axial heterogenous hyperdense mass lesion with significant peri-lesional edema and midline shift. MRI images (B-F), B (Sagittal T1), C (Axial T2), D (Coronal FLAIR), E (Post Contrast), F (Gradient sequence) showing a large heterogenous signal intensity intra-axial lesion in left parietal region in para-falcine location. Lesion shows heterogeneous post contrast enhancement with central non enhancing area suggesting necrosis. There is blooming on gradient sequence suggestive of hemorrhage. Significant peri-lesional edema and midline shift noted.

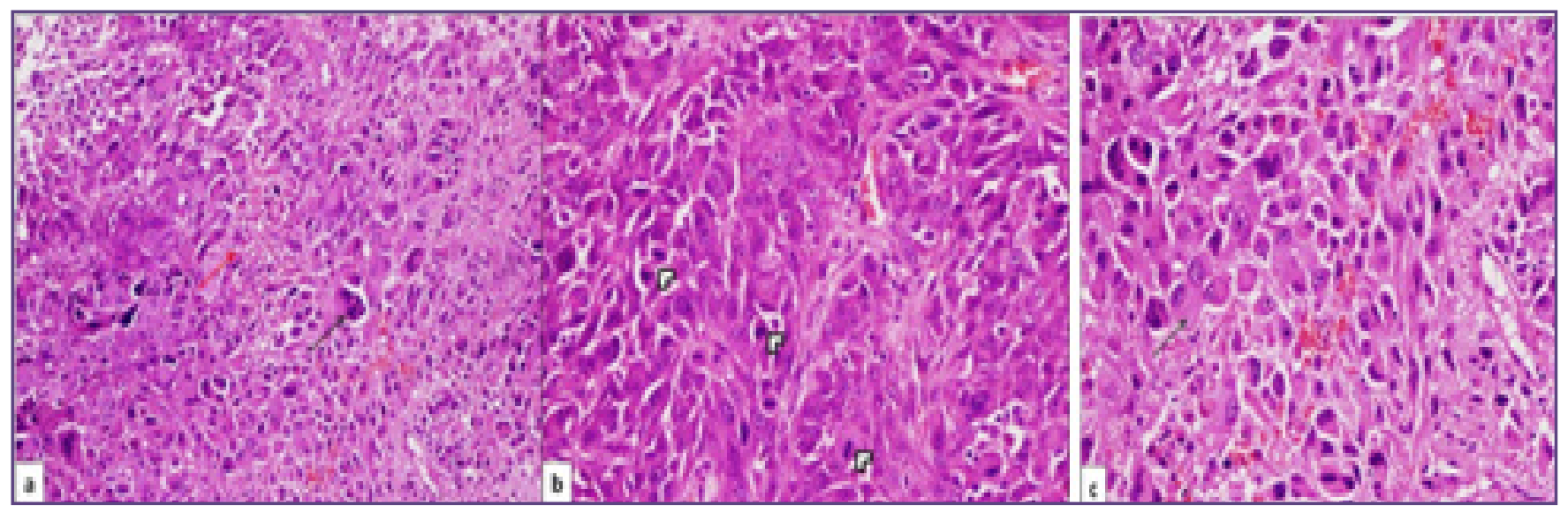

Fig. 2: Photomicrographs showing a) A tumor comprising of large epithelioid cells arranged in sheets with area of necrosis (red arrow) and frequent bi and multi-nucleation (black arrow) (HEx100), b) Many atypical mitotic figures (arrow heads) (HEx200) and c) The cells displaying eccentric nuclei with vesicular chromatin, prominent nucleolei and abundant amount of eosinophilic cytoplasm (arrow) (HEx200). 


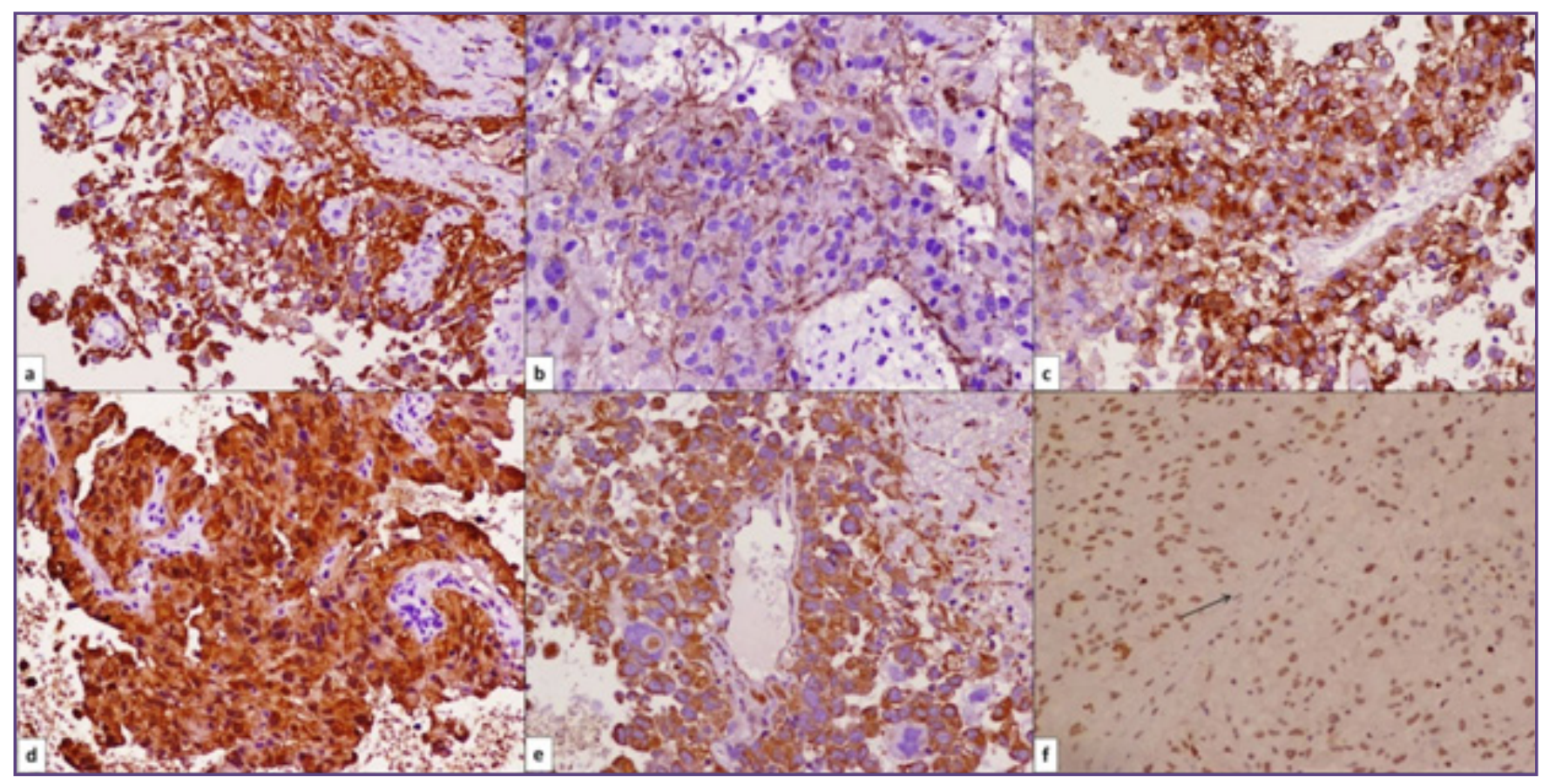

Fig. 3: The photomicrographs showing immunohistochemical expression in tumor cells a) GFAP diffuse cytoplasmic and fibrillary (IHCx200), b) CK20 membranous (IHCx200), c) EMA membranous (IHCx200), d) S-100 both nuclear and cytoplasmic staining (IHCx200), e) vimentin cytoplasmic (IHCx200) and retention of INI-1 positivity (arrow showing positive internal control in endothelial cell) (IHCx200).

\section{References}

1. Louis DN, Perry A, Reifenberger G, von Deimling A, Figarella-Branger D, Cavenee WK et al. The 2016 World Health Organization Classification of Tumors of the Central Nervous System: a summary. Acta Neuropathol. 2016;131(6):803-20.

2. Tanaka S, Nakada M, Hayashi Y, Nakada S, SawadaKitamura S, Furuyama N, et al. Epithelioid glioblastoma changed to typical glioblastoma: the methylation status of MGMT promoter and 5-ALA fluorescence. Brain Tumor Pathol. 2011;28(1):59-64.

3. Kleinschmidt-DeMasters BK, Aisner DL, Birks DK, Foreman NK. Epithelioid GBMs show a high percentage of BRAF V600E mutation. Am J Surg Pathol. 2013;37(5):685-98.

4. Kleinschmidt-DeMasters BK, Alassiri AH, Birks DK, Newell KL, Moore W, Lillehei KO. Epithelioid versus rhabdoid glioblastomas are distinguished by monosomy 22 and immunohistochemical expression of INI-1 but not claudin 6. Am J Surg Pathol. 2010;34(3):341-54.

5. Stupp R, Mason WP, van den Bent MJ, Weller M, Fisher B, Taphoorn MJ et al. European Organisation for Research and Treatment of Cancer Brain Tumor and Radiotherapy Groups. National Cancer Institute of Canada Clinical Trials Group. Radiotherapy plus concomitant and adjuvant temozolomide for glioblastoma. N Engl J Med. 2005;352(10):987-96.

6. Alexandrescu S, Korshunov A, Lai SH, Dabiri S, Patil S, Li R et al. Epithelioid Glioblastomas and Anaplastic Epithelioid Pleomorphic Xanthoastrocytomas-Same Entity or First Cousins? Brain Pathol. 2016;26(2):215-23.

7. Tanaka S, Nakada M, Nobusawa S, Suzuki SO, Sabit H, Miyashita $\mathrm{K}$ et al. Epithelioid glioblastoma arising from pleomorphic xanthoastrocytoma with the BRAF V600E mutation. Brain Tumor Pathol. 2014;31(3):172-6.

*Corresponding author:

Dr Rakesh Kumar Gupta, Senior Resident, Department of Pathology, G B Pant Institute of Postgraduate Medical Education and Research, New Delhi -110002 Phone: +91 9718599076

Email: rakesh.newjobi@gmail.com

Financial or other Competing Interests: None.

Date of Submission : 14.03.2017

Date of Acceptance : 12.04.2017

Date of Publication : 28.05.2017 